\title{
A Case of Abdominal Pain, Hypoxia and Clubbing
}

\author{
Jennifer N. Stojanª, b, Jeffrey M. Rohde ${ }^{a}$, Michael Lukela ${ }^{a, b}$
}

\begin{abstract}
Cyanotic congenital heart disease is often diagnosed in infancy. Here we present a case of a 19-year-old female who presented with abdominal pain, hypoxia and clubbing. She was found to have a splenic infarct on CT scan. The splenic infarct was caused by an emboli from the right side of the circulation that traveled across a communication between the right pulmonary artery and the left atrium. This lesion has only been cited in 23 adult cases [1]. When the communication was identified on transthoracic echocardiogram, she was diagnosed with cyanotic congenital heart disease and the etiology for her clubbing was discovered.
\end{abstract}

Keywords: Abdominal pain; Hypoxia; Clubbing; Cyanotic congenital heart disease; Splenic infarct

\section{Introduction}

Although, abdominal pain is a common chief complaint for a general medicine patient, rarely is a splenic infarct the etiology. The treatment for a splenic infarct is often supportive, but clinicians must continue to search for an underlying etiology as the infarct may be a presenting symptom of a serious underlying condition. We report a case of splenic infarct caused by a direct communication between the right pulmonary artery and the left atrium. To date only 23 cases of such a lesion exist in the literature in adult patients [1].

Manuscript accepted for publication November 16, 2012

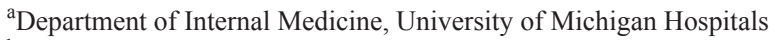

${ }^{\mathrm{b}}$ Department of Pediatrics and Communicable Diseases, University of Michigan Hospitals

${ }^{\mathrm{c}}$ Corresponding author: Jennifer N. Stojan, Institution: University of Michigan, 3119 Taubman Center, SPC 5376, 1500 East Medical Center Drive, Ann Arbor, MI 48109, USA. Email: jstojan@umich.edu

doi: http://dx.doi.org/10.4021/jmc966w

\section{Case Report}

A 19-year-old woman with a history of migraines, depression, and persistent dyspnea presented to the emergency department with abdominal pain. Three days prior to presentation, she acutely developed pleuritic, left upper quadrant abdominal pain that radiated to her left shoulder. This was not associated with nausea, vomiting, diarrhea, change in appetite, or fevers. She denied trauma, cough, recent travel, or worsening of her chronic dyspnea. She was a life-long nonsmoker and did not use oral contraceptives. There was no family history of clotting disorders.

On physical examination, the patient was afebrile and in mild distress from her abdominal pain. Her respiratory rate was 18 breaths per minute, heart rate 124 beats per minute, and blood pressure $111 / 50 \mathrm{mmHg}$. Her oxygen saturation was $84 \%$ breathing room air. Her lungs were clear to auscultation bilaterally. Cardiac examination revealed a grade $2 / 6$ systolic murmur loudest at the left mid-sternal border radiating to her back. Her abdomen was soft with normal active bowel sounds, but was tender to palpation in the left upper quadrant without guarding or rebound. She did not have

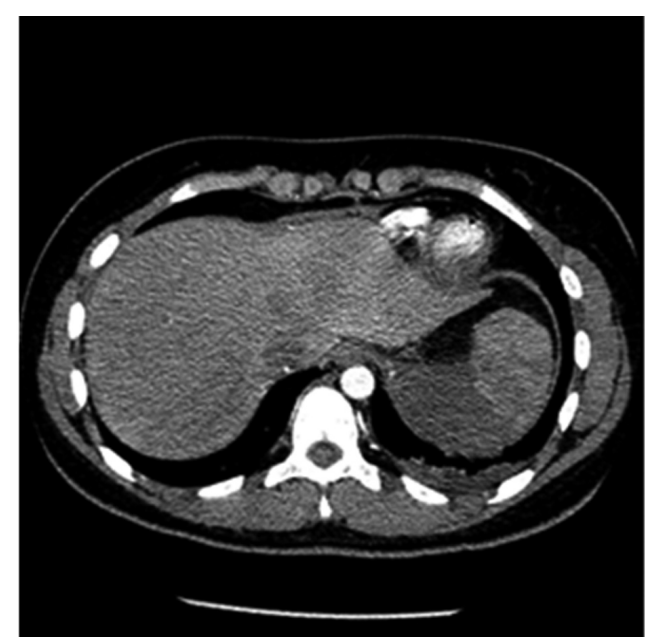

Figure 1. CT scan showing a wedge shaped territory of lowattenuation within the spleen concerning for a splenic infarct. 


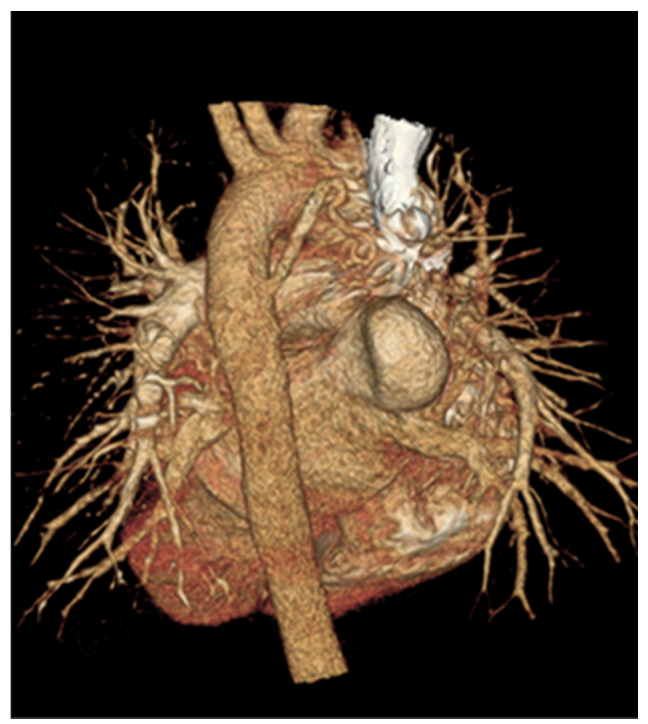

Figure 2. Reformatted PE CT showing a direct communication between the right pulmonary artery and the left atrium.

associated hepatosplemegaly. Symmetric clubbing and nail bed cyanosis were noted in her fingertips. Laboratory testing showed a hemoglobin of $11.8 \mathrm{mg} / \mathrm{dL}$ and a white blood cell count of $9.3 \mathrm{k} / \mathrm{mm}^{3}$. Electrolytes, renal function, liver function tests, amylase, and lipase were normal. Urinalysis and urine pregnancy test were negative. An arterial blood gas was recommended which the patient refused. A pulmonary embolism protocol computed tomography (CT) of the chest showed no evidence of pulmonary embolism, but demonstrated left atrial enlargement and a splenic infarct (Fig. 1). Based on the patient's longstanding history of dyspnea and findings on physical examination including hypoxemia, cardiac murmur, and the presence of clubbing and cyanosis in her fingertips there was strong suspicion that a right to left shunt allowed a paradoxical embolus led to her splenic infarction. Three-dimensional formatting of her pulmonary embolism protocol CT showed a direct communication between the right pulmonary artery and the left atrium (Fig. 2 ), which was confirmed by transthoracic echocardiogram (Fig. 3).

The patient's abdominal pain, caused by her splenic infarct, was treated symptomatically and resolved without complication. She was treated empirically with enoxaparin to prevent recurrence of thromboembolic events. She ultimately underwent surgical ligation of her congenital fistula with resolution of her shunt and correction of her hypoxemia and dyspnea.

\section{Discussion}

Splenic infarction occurs when there is occlusion of the arterial or venous circulation of the spleen resulting in splenic parenchymal ischemia. Patients may be asymptomatic or present with fever, chills, nausea, vomiting, left upper quadrant, flank, back or shoulder pain. There may be abdominal tenderness or splenomegaly on exam [2]. Routine laboratory studies are not specific to splenic infarction, but often demonstrate leukocytosis and elevation in lactate dehydrogenase. CT findings may show a well-defined area of decreased attenuation within the splenic parenchyma that does not enhance with contrast. The management of splenic infarcts is primarily supportive with pain medications used to

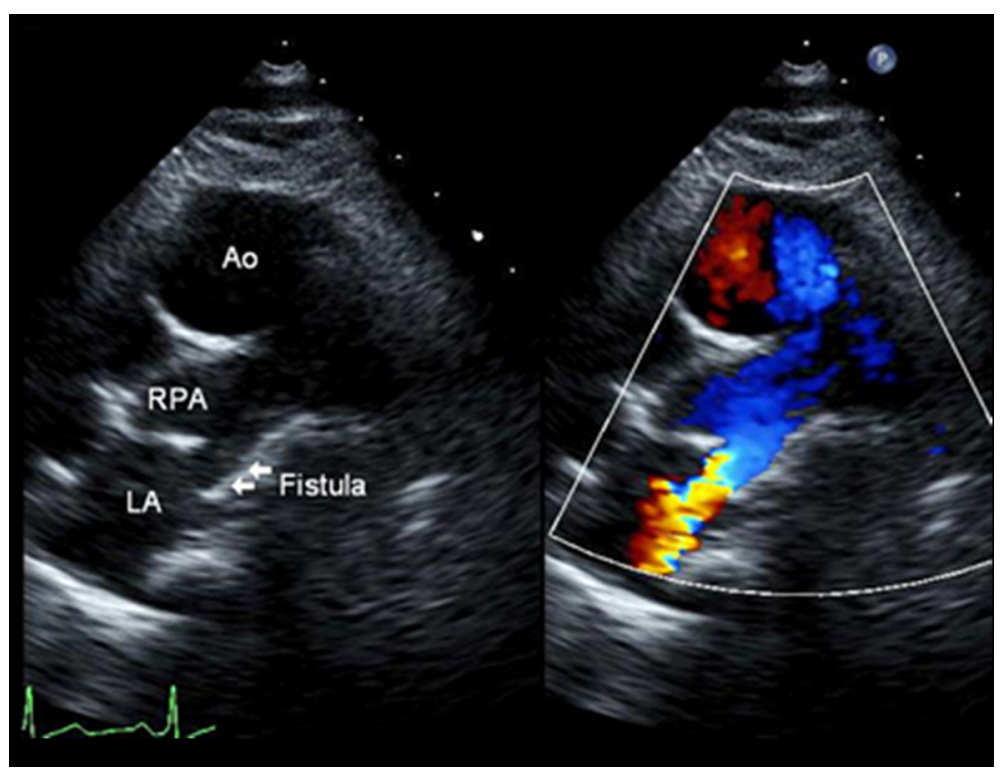

Figure 3. Transthoracic echocardiogram demonstrating a moderate to large fistulous communication between the right pulmonary artery and the left atrium. 
treat the associated discomfort. Rarely, surgical intervention is required when complications such as splenic rupture, abscess formation, or worsening hypersplenism arise requiring splenectomy in $4 \%$ of patients [3].

Although the management of splenic infarcts is often supportive, it is essential for clinicians to search for an underlying process when the diagnosis is made as it may be the presenting symptom of a serious underlying condition [3]. Potential etiologies of splenic infarction include hypercoagulable states, malignancy, hemoglobinopathy, myeloproliferative disorders, or thromboembolic disease, such as atrial fibrillation, endocarditis, or left ventricular thrombus following myocardial infarction. Although emboli are more likely to originate from the left side of the circulation, they may originate from the right side if a direct communication exists such as a patent foramen ovale or, as in this case, congenital heart disease.

As it relates to our patient, who presented with the classic findings of clubbing and hypoxemia in the setting of a heart murmur, only 82 cases have been reported in the literature describing a direct communication between the right pulmonary artery and the left atrium. Of these cases only 23 were diagnosed in adults [1]. Cyanotic congenital heart disease is rarely diagnosed in adults, but carries a five-year mortality of $12.6 \%$ [4]. Complications from untreated cyanotic congenital heart disease include erythrocytosis, problems with hemostasis, hyperuricemia, renal dysfunction, cerebrovascular accidents, endocarditis, brain abscesses, supraventricular and ventricular arrhythmias, and the development of Eisenmenger's Syndrome [3, 5-8]. Female patients may experience a delay in menarche or menorrhagia and if they chose to become pregnant, pregnancies may result in a high incidence of miscarriage, premature births or low birth weight babies $[9,10]$. If not repaired, the $2008 \mathrm{ACC} / \mathrm{AHA}$ guidelines recommend prophylaxis for the prevention of endocarditis and the avoidance of competitive sports [5].

\section{Authors Contributions}

Dr. Stojan identified the case described, wrote the draft of the manuscript, and revised the manuscript into its final form; Drs. Rohde and Lukela reviewed the drafting of the article, providing revisions for important intellectual content. All authors fulfill the authorship criteria required by the Journal.

\section{Grant Support}

There was no grant supporting the preparation of this manuscript.

\section{Conflict of Interest}

All authors declare that there is no conflict of interest and there are no financial disclosures related to this case.

\section{References}

1. Luo GH, Ma WG, Huang LJ, Yan J, Zhu XD. Surgical and transcatheter closure of right pulmonary artery to left atrial fistula. J Card Surg. 2011;26(2):130-134.

2. Lawrence YR, Pokroy R, Berlowitz D, Aharoni D, Hain D, Breuer GS. Splenic infarction: an update on William Osler's observations. Isr Med Assoc J. 2010;12(6):362365.

3. Antopolsky M, Hiller N, Salameh S, Goldshtein B, Stalnikowicz R. Splenic infarction: 10 years of experience. Am J Emerg Med. 2009;27(3):262-265.

4. Engelfriet P, Boersma E, Oechslin E, Tijssen J, Gatzoulis MA, Thilen U, Kaemmerer H, et al. The spectrum of adult congenital heart disease in Europe: morbidity and mortality in a 5 year follow-up period. The Euro Heart Survey on adult congenital heart disease. Eur Heart J. 2005;26(21):2325-2333.

5. Warnes CA, Williams RG, Bashore TM, Child JS, Connolly HM, Dearani JA, del Nido P, et al. ACC/AHA 2008 Guidelines for the Management of Adults with Congenital Heart Disease: a report of the American College of Cardiology/American Heart Association Task Force on Practice Guidelines (writing committee to develop guidelines on the management of adults with congenital heart disease). Circulation. 2008;118(23):e714-833.

6. Ammash N, Warnes CA. Cerebrovascular events in adult patients with cyanotic congenital heart disease. J Am Coll Cardiol. 1996;28(3):768-772.

7. Ware JA, Reaves WH, Horak JK, Solis RT. Defective platelet aggregation in patients undergoing surgical repair of cyanotic congenital heart disease. Ann Thorac Surg. 1983;36(3):289-294.

8. Ross EA, Perloff JK, Danovitch GM, Child JS, Canobbio MM. Renal function and urate metabolism in late survivors with cyanotic congenital heart disease. Circulation. 1986;73(3):396-400.

9. Drenthen W, Hoendermis ES, Moons P, Heida KY, RoosHesselink JW, Mulder BJ, Van Dijk AP, et al. Menstrual cycle and its disorders in women with congenital heart disease. Congenit Heart Dis. 2008;3(4):277-283.

10. Presbitero P, Somerville J, Stone S, Aruta E, Spiegelhalter D, Rabajoli F. Pregnancy in cyanotic congenital heart disease. Outcome of mother and fetus. Circulation. 1994;89(6):2673-2676. 